\title{
ON THE INTERCHANGE OF LIMIT AND LEBESGUE INTEGRAL FOR A SEQUENCE OF FUNCTIONS*
}

\author{
BY \\ T. H. HILDEBRANDT
}

R. L. Jeffery in a paper on The integrability of a sequence of functions $†$ has given a number of necessary and sufficient conditions for $\lim _{n} \int_{E} f_{n}=\int_{E} F$, where $f_{n}$ and $F$ are summable on the measurable set $E$ and $\lim _{n} f_{n}=F$ on $E$. The object of this note is to give an additional condition for the validity of this interchange, embodied in the

THEOREM. If $U(n, \delta)$ is the least upper bound, and $L(n, \delta)$ is the greatest lower bound of $\int_{e} f_{n}$ for all measurable subsets $e$ of $E$ for which me $\leqq \delta$, then a necessary and sufficient condition that $\lim _{n} \int f_{n}=\int F$ on $E$, is that

$$
\lim _{\delta \rightarrow 0} \lim _{n \rightarrow \infty}[U(n, \delta)+L(n, \delta)]=0 .
$$

We are using the notation $\lim _{\delta} \lim _{n}$ in the standard sense of the common value of $\lim _{\delta} \underline{\lim }_{n}$ and $\lim _{\delta} \varlimsup_{n}$. In $(\epsilon, \delta)$ form the condition of the theorem is equivalent to the following: $\neq$ For every $\epsilon>0$ there exists a $\delta_{\epsilon}$ such that for every $\delta \leqq \delta_{\epsilon}$, there exists an $n_{\delta \epsilon}$ such that if $n \geqq n_{\delta \epsilon}$, then $|U(n, \delta)+L(n, \delta)| \leqq \epsilon$.

We establish the equivalence of our condition with the necessary and sufficient condition I of Jeffery, viz.

$$
\lim _{l \rightarrow \infty} \int_{C(l, \eta)} f_{n}=0 \quad(n \geqq l)
$$

where $C(l, \eta)$ is the complement relative to $E$ of the set for which

for every $n \geqq l$.

$$
\left|f_{n}-f\right| \leqq \eta
$$

By the definition of $U(n, \delta)$, for every $\epsilon$ and $\delta$, there exists a subset $e$ of $E$ of measure less than $\delta$ such that

$$
U(n, \delta)-\epsilon \leqq \int_{e} f_{n} \leqq U(n, \delta) .
$$

Since $U(n, \delta) \geqq 0$, we can obviously assume that $f_{n} \geqq 0$ on $e$. Now for any $l$ and $\eta$

* Presented to the Society, December 31, 1930; received by the editors December 1, 1930.

$\dagger$ Cf. the present number of these Transactions.

$\ddagger$ Cf., for instance, Hildebrandt, Note on interchange of limits, Bulletin of the American Mathematical Society, vol. 34 (1928), p. 80 . 


$$
\left|\int_{e} f_{n}-\int_{e C(l, \eta)} f_{n}\right| \leqq \int_{e-e C(l, \eta)}\left|f_{n}-F\right|+\int_{e-e C(l, \eta)}|F| \text {. }
$$

As a consequence if we take $\delta$ so that $\delta \eta \leqq \epsilon / 2$ and so that, for $m e \leqq \delta$, it is true that $\int_{\odot}|F|<\epsilon / 2$, then provided $n \geqq l$

$$
\left|\int_{0} f_{n}-\int_{e C(l, \eta)} f_{n}\right| \leqq \epsilon .
$$

Let $C^{+}$be the subset of $C(l, \eta)$ for which $f_{n} \geqq 0$, and $C^{-}$the set for which $f_{n}<0$. If then $l$ be chosen so that $m C(l, \eta) \leqq \delta$, and $n \geqq l$, then

$$
U(n, \delta)-2 \epsilon \leqq \int_{e C(l, \eta)} f_{n}=\int_{e C^{+}} f_{n} \leqq \int_{C^{+}} f_{n} \leqq U(n, \delta) .
$$

In a similar way we show that

$$
L(n, \delta) \leqq \int_{C^{-}} f_{n} \leqq L(n, \delta)+2 \epsilon .
$$

Hence if $\delta \leqq \delta_{\text {e, }}$ and $l \geqq l_{\delta \text { e }}$, with $n \geqq l$, we have

$$
\left|\int_{C(l, \eta)} f_{n}-[U(n, \delta)+L(n, \delta)]\right| \leqq 2 \epsilon .
$$

From this statement follows the equivalence of the conditions

$$
\lim _{l \rightarrow \infty} \int_{C(l, \eta)} f_{n}=0(n \geqq l) \text { and } \lim _{\delta \rightarrow 0} \lim _{n \rightarrow \infty}[U(n, \delta)+L(n, \delta)]=0 .
$$

In so far as

$$
\lim _{\delta \rightarrow 0}[U(n, \delta)+L(n, \delta)]=0
$$

for every $n$, our condition is equivalent to the equality of the iterated limits $\lim _{\delta} \lim _{n}$ and $\lim _{n} \lim _{\delta}$ of the function $U(n, \delta)+L(n, \delta)$. Since the existence

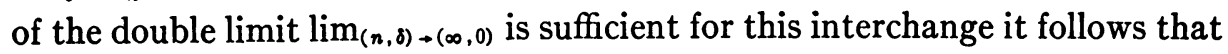

$$
\lim _{(n, \delta) \rightarrow(\infty, 0)}[U(n, \delta)+L(n, \delta)]=0
$$

is also a sufficient condition for $\lim _{n} \int_{E} f_{n}=\int_{E} F$. That it is not necessary follows from the example given by Jeffery in $\$ 3$.

If the integrals $\int f_{n}$ are equicontinuous on $E$, i.e., if

$$
\lim _{m e \rightarrow 0} \int_{e} f_{n}=0
$$


uniformly in $n$, then obviously $\lim _{\delta}[|U(n, \delta)|+|L(n, \delta)|]=0$, uniformly in $n, U$ and $L$ being even taken with respect to any subset $e$ of $E$. Applying the standard theorem on interchange of iterated limits, we get at once the Vitali theorem for all subsets $e$ of $E$. On the other hand, if all the functions $f_{n}$ are positive on $E$, then $U(n, \delta)$ converges to zero monotonically in $\delta$, while $L(n, \delta)=0$. By applying the following generalization of Dini's Theorem:*

If $\lim _{n} \lim _{\delta} U(n, \delta)=\lim _{\delta} \lim _{n} U(n, \delta)$, and $U(n, \delta)$ is monotone in $\delta$, then $\lim _{\delta} U(n, \delta)$ exists uniformly in $n$,

we find the well known result that when $f_{n} \geqq 0$, equicontinuity of $\int f_{n}$ is a necessary condition for $\lim _{n} \int f_{n}=\int F$ on $E$.

The theorem of this note is still valid if the convergence of $f_{n}$ to $f$ on $E$ is convergence in a measure, $\dagger$ i.e., if $D(n, \eta)$ is the set of points of $E$ for which $\left|f_{n}-f\right|>\eta$ then $\lim _{n} m D(n, \eta)=0$ for each $\eta$. Jeffery's Theorem must be altered so that the condition becomes

$$
\lim _{n} \int_{\nu(n, \eta)} f_{n}=0
$$

for every $\eta$. The proofs for this more general case require only slight changes from those given.

* Cf. Bulletin of the American Mathematical Society, vol. 21 (1914), p. 113.

$\dagger$ The possibility of this extension was suggested by L. M. Graves.

UNIVERSITY OF Michigan, ANN ARBor, Mich. 